\title{
Evaluating the Standard Assumptions of Demand Planning and Control
}

\author{
Peter Nielsen and Kenn Steger-Jensen \\ Aalborg University, Department of Production, Fibigerstraede 16, 9220 \\ Aalborg Oest, Denmark, \{peter;kenn\}@production.aau.dk
}

\begin{abstract}
This paper investigates customer ordering behavior and compares a particular instance to the standard assumptions of demand planning and control. Experience shows that the performance of these planning processes often do not match what could be expected. Based on a presented case, implications for demand planning performance are inferred. This leads to an analysis tool for diagnosing potential problems in the demand planning approach.
\end{abstract}

\section{Keywords}

Demand planning and control, numerical study, performance impact

\section{Introduction}

Demand planning systems are a vital part of the business structure of companies. More so in manufacturing companies depending on a demand planning system to deliver feasible plans ensuring the best (or close to) utilization of resources while adhering to material, resource and due date constraints. Today most companies use some variant of the hierarchical demand planning approach presented by Hax and Meal [1]. However the approach's success depends on the conformity to a number of assumptions regarding independence of planning objects' (items, product families, resources) behavior and the ability to aggregate and disaggregate information. This subject has been studied in detail (among others: Axsäter [2]). However no method has been presented that accurately predicts the consequences of deviating from the inherent standard assumptions. This paper suggests a method to evaluate the performance of the demand planning processes and establish the potential impact of deviating from the standard assumptions. The focus is on downstream activities and consequently upstream constraints and impacts are not considered.

This paper contains four distinct parts. First, a literature review, discussing issues within demand planning and control, is presented. Second, results from a study of 
demand patterns, in a company using a mix of ATO and MTS, are presented. Third, a discussion of the implications for demand planning performance and processes is given, before finally a conclusion is reached.

\section{The demand planning connection}

When looking at demand planning in an manufacturing environment two main aspects naturally need to be taken into account: materials and resources. The relative importance of these two areas will reflect the competitive priorities and market conditions of the particular company. The two are typically manifested through demand planning and subsequently for materials as inventory management. Within inventory management Silver [3] state three statistical assumptions: 1) assuming a particular demand pattern, 2) assuming that parameters of the distribution are known and 3) assuming stationarity. To these assumptions an equally important assumption must be added, namely the assumption of independency of parameters. Although much has changed since Silver statements in 1981, the underlying assumptions have not changed significantly. Demand planning is often implemented as hierarchical demand planning (HDP) $[1,4,5]$. The main objectives of HDP is to level production output thereby easing allocation of resources and materials. Ideally aggregation naturally levels demand and reduces noise. However to successfully achieve a reduction of noise Sales and Operations Planning must be integrated in the Master Production Scheduling process. In practice only the ideal situation is considered. This coincides with the assumption that if HDP is to work, the aggregate demand plan must successfully be disaggregated to a number of feasible plans [6]. The assumption is a one-toone relationship in aggregation and disaggregation [1,2]. As a result order size, timing of orders, variance in order size and the number of orders are all assumed to be stochastic and independent. Demand is furthermore often assumed to be seasonal fulfilling one of the main advantages of HDP; namely leveling [5,7]. The interdependency (or lack hereof) between planning parameters is crucial for the performance of the demand planning system in both an ATO and MTS environment.

The major potential pitfall in HDP is of course the ability to aggregate and disaggregate data and plans. To aggregate and disaggregate a dimension of similarity between planning objects is needed. As a result products are typically grouped by similarity of BOM's, while manufacturing resources are grouped by manufacturing capabilities. To justify grouping items in this manner one must assume that they have similar demand patterns and similar use of manufacturing resources $[1,5]$. However similarities of BOM's does not necessarily mean that the grouped items have similar seasonality (as assumed) or load on resources. The tendency is to tie the underlying data model inexplicitly to materials via the BOM and only connect capacity through a secondary check for feasibility of plans [8]. However this one dimensional approach seems inappropriate in an ATO environment where planning of resources precedes material planning in importance. Another important limitation of HDP is the need to partition demand into time buckets / periods. The size of these time buckets to a large extend determines the degrees of freedom in the demand planning and control system. The underlying assumption is that demand, although stochastic, 
occurs at a steady rate during the period [8] i.e. that orders are uniformly distributed. Systematic divergence from this assumption gives suboptimal performance of lead times (in ATO) and service level (MTS with multiple replenishments per period) or excessive safety stock (MTS single replenishment per period). This leads to a general problem since the length of the periods is often uncritically chosen as a month due to e.g. the customer credit period, the manner in which forecasts are made or the ability to adjust manufacturing capacity. The demand pattern seldom enters into the equation.

The hypothesis in this paper is that the standard assumptions seldom hold true and that subsequently the demand planning process suffers poorer than expected performance. In this paper an analysis of demand patterns, in a particular case company using a mix of ATO and MTS, is presented. The hypothesis is that the demand pattern in the company deviates significantly from the standard assumptions giving poor performance regarding on-time-delivery, lead times and service level.

\section{Results}

To investigate whether this hypothesis holds true a method for analysis was developed. The following example focuses on numerically evaluating demand patterns of three products from a major product family over a period of three years. The products are all produced in the same manufacturing cell, but have varying impact on resource load. The products have $80-85 \%$ similar components and constitutes approximately $75 \%$ of the total demand of the product family. Demand for products 1 and 2 can be assumed to be stationary and normally distributed. Demand for product 3 is somewhat more erratic and does not follow any standard distribution, nor did it exhibit any significant trend. Product 1 is often sold with a combination of other products, making it critical from a demand planning perspective. On the surface a textbook case for the use of HDP. However in practice the company frequently experience problems fulfilling orders on time and unexpected underutilization of resources. To investigate, the following demand pattern parameters were studied: variance of order size within a period, mean order size within a period, the no. of orders per period and the total demand per period. These parameters were checked for correlation within the individual products first. The results are shown in table 1.

The results show that the mean order size and the number of orders for a period are negatively correlated $(-0.55)$ for product 1 . This indicates that as the number of orders increase the order size decreases, resulting in more changeovers. This causes problems in the ATO part of the company since a relatively large changeover is incurred between assemblies. Products 2 and 3 show no connection between the number of orders and the mean order size. The standard assumption would be no correlation between the mean order size and the number of orders.

It can be seen from table 1 that the number of orders is positively correlated $(0.53$ - 0.67) to total demand for all three products included in the study. A positive correlation indicates that as the total demand increases the number of orders also increase. However the parameters are not perfectly correlated, thereby breaking the assump- 
tion of a constant ratio of orders compared to total demand. As changeovers are nontrivial this impacts the performance of the aggregate capacity plan.

Table 1. Correlation between different demand pattern parameters.

\begin{tabular}{|c|c|c|c|}
\hline Product 1 & Variance of order size & Mean order size & No. of orders \\
\hline Mean order size & 0.78 & & \\
\hline No. of orders & -0.26 & -0.55 & \\
\hline Total demand & 0.55 & 0.31 & 0.53 \\
\hline \multicolumn{4}{|l|}{ Product 2} \\
\hline Mean order size & 0.75 & & \\
\hline No. of orders & 0.13 & -0.01 & \\
\hline Total demand & 0.66 & 0.69 & 0.66 \\
\hline \multicolumn{4}{|l|}{ Product 3} \\
\hline Mean order size & 0.77 & & \\
\hline No. of orders & 0.08 & 0.21 & \\
\hline Total demand & 0.56 & 0.82 & 0.67 \\
\hline
\end{tabular}

In an ATO environment the relative size of orders impacts the ability to shift production focus. As a consequence, the variance of order size in a period can be used as a yardstick for the required product mix flexibility. The variance of order size is clearly correlated to total demand and mean order size. Both relationships can be expected, however the correlation to total demand offers some problems. This connection indicates as the total demand increases so does the diversity of order sizes. This would indicate that the manufacturing planning and control system needs greater flexibility when demand is high. This can necessitate a shift from a leveling strategy to a chase strategy. In ATO companies order sizes should not differ between points of time in any systematic manner. To test this, a combined density function for order size and time using the kernel method for multivariate data [9], is developed. The kernel used is the standard bivariate normality density function [9]. To avoid over fitting, the model is fitted to the relative to mean order size per day a month as the periods currently used are one month. Subsequently the largest probable relative order size is calculated for each day. Products 1 and 3 show no clear preference for large/small orders at any given time. This is however not the case for product 2. A graph of the largest expected relative order size for product 2 is shown in figure 1.

From figure 1 it can be seen that large orders seem to occur within rather narrow timeframes of the month. In particular days 8-12 and 22-25 tend to be preferred dates for large orders. In an ATO environment lead time is typically an important competitive priority. Since these products are sold as ATO, spikes in the order size stresses the demand planning system, resulting in increased lead times and occasional use of overtime. In practice this means that at certain recurring points of time a chase strategy must be adopted instead of leveling. If the demand pattern was found in a MTS environment instead, choosing an inappropriate inventory management techniques would lead either to poor service level or low inventory turnover. Consider a con- 
tinuous review technique: If large orders occur at specific times the probability for stock out will be disproportional large at/after these times.

Figure 1. Largest expected relative order size at a given time for different probabilities.

\section{Isoprobability lines for occurrences of order sizes}

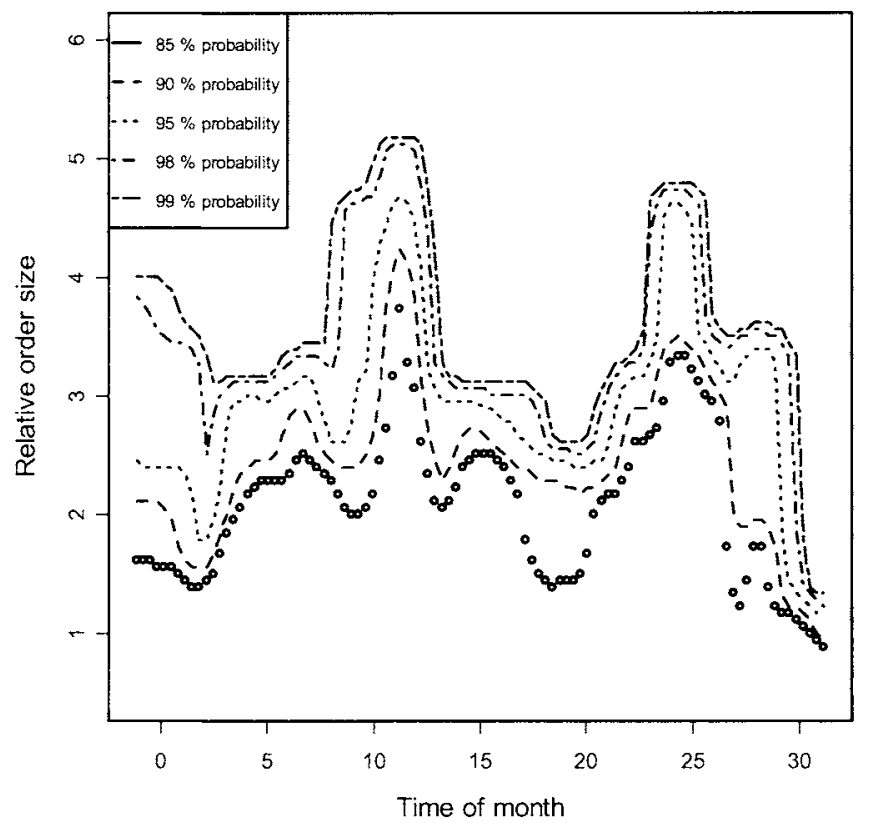

After examining the demand pattern individually, the four parameters were also checked between products, see table 2 for a brief summary.

Table 2. Correlation of demand pattern parameters between products.

\begin{tabular}{|l|c|}
\hline Interproduct correlation: & Range of correlation between products: \\
\hline Variance of order size & $0.13-0.30$ \\
\hline Mean order size & $0.24-0.39$ \\
\hline No. of orders & $0.05-0.38$ \\
\hline Total demand & $0.20-0.30$ \\
\hline
\end{tabular}

From table 2 it is evident that it can be assumed that the demand patterns of the three products are noncorrelated for total demand and variance of order size i.e. they experience no evidence of similar seasonality. This was also supported since a test of autocorrelation of all parameters for all products with a lag of 1-12 periods, showed no significant correlation between periods. However some indication of a connection between the order size and the number of orders per period, does seem to be present. In an ATO environment this gives some problems, since a positive correlation indi- 
cate that an increase in the number of orders for one product coincides with an increase in the number of orders for another product. This stresses the manufacturing system and violates the basic assumption of independence that enables leveling.

The findings indicate that the assumption of independence of individual demand pattern parameters is incorrect and subsequently poor performance of the demand planning processes will be expected.

\section{Implications}

This paragraph includes an overview of the cause and effects between indicators and demand planning assumptions. Subsequently a number of Key Indicators (KI's) and their values, if conforming to standard assumptions of demand planning, is presented. This can be used to diagnose the state of a given demand planning system. Finally avenues of further research are presented.

\subsection{Causality}

Based on the assumptions of HDP and inventory management techniques in general and the case study, the following steps forms a prudent method for analysis:

1. Identify poorly performing planning areas.

- These areas will have lower/higher than expected service level or utilization.

2. Identify critical products or resources - i.e. choose the defining dimension for the data model.

- A multi criteria $\mathrm{ABC}$ analysis [10] could be conducted. The criteria could be: materials (volume, cost, similarity of BOM, seasonality, etc.) and resources (capacity, similarity, etc.)

3. Analyze the demand pattern parameters for the individual critical products.

- Variance of order size, Mean order size, No. of orders and Total demand.

- Distribution of demand within periods e.g. Kolmogorov-Smirnov test for uniformity of demand rate.

4. Analyze the interaction of demand pattern parameters between critical products.

- Variance of order size, Mean order size, No. of orders and Total demand

5. Establish where (if any) deviations from the standard assumptions exists.

- Take corrective actions i.e. adjust the demand planning approach accordingly.

The method presented above is still bound to the data model inherent in HDP i.e. depends on the materials and BOM. The method can be retooled to focus on load of resources, however the data has not been available for this in the current study.

Based on the analysis, a number of KI's for demand pattern parameters can be found. The expected values are presented in table 3 .

The KI's presented in table 3, offer the theoretical expected values for correlation between demand pattern parameters for individual items, but not limits on what constitutes a significant deviation from the assumptions. The limits will be situational and depend on the specific interdependencies in a given company. If several of the parameters deviate simultaneously the negative impact on performance is enhanced. 
Table 3. Key Indicators for demand pattern parameters and expected values in HDP.

\begin{tabular}{|l|c|c|c|}
\hline Product 1 & Variance of order size & Mean order size & No. of orders \\
\hline Mean order size & $0-1.00$ & & \\
\hline No. of orders & 0 & 0 & \\
\hline Total demand & 0 & 0 & 1.00 \\
\hline
\end{tabular}

For the interproduct relationships varying behavior can be expected. If the same seasonality or trend applies for two products the total demand per period will be correlated between products. However if no similar trend or seasonality is present, the demand should be negatively correlated to ensure that leveling is advantageous.

Applying the method involves 1) conducting the analysis and 2) using the results to develop a holistic forecasting and planning method taking the covariance of critical control parameters into account. A demand planning system should be able to handle deviations from the standard assumptions. The consequence is that not only total demand per period needs to be forecasted, but also the characteristics of the demand pattern. Consequently a density function describing several dimension, e.g. order sizes, order frequency, etc., and not just demand rate, should be used, depending on the requirements of the planning process.

An issue that has not been resolved is the impact of using fixed time buckets. The enriched demand pattern models suggested above, still depend on fixed length periods. A potential solution is to use autocorrelation to establish when/whether the demand pattern parameters exhibit systematic behavior. If a systematic ordering pattern is present, then autocorrelation could be used to identify it and subsequently the size of the time buckets can be established. Although it would complicate demand planning further, the path to better demand planning processes could be to dynamically resize time buckets and continually fit demand patterns to these time buckets.

When looking at the suggested method for analysis an issue arises. To get the method to work, a significant amount of data is needed. This means the method in present form only can be applied to products were a substantial ordering history has been established. The dependency on historical data can not be avoided, since it is in the nature of demand planning to - at least to some extend - depend on historical data. Furthermore one can argue that for problems to arise in a demand planning process they must be recurring over time, enabling at least some numerical analysis.

\subsection{Further research}

To evaluate the impact of deviating from the standard assumptions, simulation studies should be conducted. These would be able to show specific ranges for the KI's presented in paragraph 4.1. This could have a large impact on the practical application of various demand planning techniques, that are often applied without studying the interdependencies of the planning objects.

The implications for practical application are daunting. Imagine demand management with knowledge of which parameters to adjust in customer ordering behavior, i.e. the order sizes, timing of orders, etc.. However it should be noted that further studies are required to validate the results and finalize a descriptive model. 


\section{Conclusion}

HDP is based on a number of standard assumptions regarding demand pattern parameters, focuses on materials and is often applied for MTS. However when looking at an ATO manufacturing environment focus should be on scheduling resources rather than on planning materials. In this paper we presents results indicating reasons for poor performance of demand planning processes. Furthermore suggestions are presented on how to amend these shortcoming of the current demand planning processes. Consequently this paper has a practical impact by giving a method for analysis of demand patterns and subsequently diagnose causes for poor performance of demand planning processes. Secondly the paper offers a theoretical solution for achieving a holistic demand planning approach not only assuming interdependencies among control parameters but including significant occurrences of covariance among parameters. The focus is on downstream influences to the demand planning processes. The presented method can with minor adjustments however be modified to address supplier behavior as well as customer ordering behavior.

\section{References}

1. C. Hax and H. C. Meal, Hierarchical Integration of Production Planning and Scheduling, in Logistics, edited by M. A. Geisler, vol. 1 of Studies in the Management Sciences, NorthHolland/American Elsevier, 1975.

2. S. Axsäter, Aggregation of Product Data for Hierarchical Production Planning, Operations Research, 29, pp. 74-756, 1981.

3. Silver, Operations Research in Inventory Management: A Review and Critique, Operations Research, 29, pp. 628-645, 1981.

4. G. R. Bitran, E. A. Haas, and A. C. Hax, Hierarchical Production Planning: A Single Stage System, Operations Research, 29, pp. 717-743, 1981.

5. C. Holt, F. Modigliani, J. Muth and H. Simon, Planning Production, Inventories and Work Force, Prentice-Hall, 1960.

6. S. Axsäter, On the Feasiblity of Aggregate Production Plans, Operations Research, 34, pp. 796-800, 1986.

7. Chung and L. J. Krajewski, Planning Horizons for Master Production Scheduling, Journal of Operations Management, 4, pp. 389-406, 1984.

8. Vollmann, W. Berry, and D. Whybark, Manufacturing Planning and Control Systems, fourth ed., Irwin/McGraw-Hill, 1997.

9. W. Silverman, Density Estimation for Statistics and Data Analysis, Chapman and Hall, 1986.

10. Flores and D. C. Whybark, Multiple Criteria ABC Analysis, International Journal of Operations \& Production Management, 6, pp. 38-46, 1986. 